\title{
Vestibular Prostheses Investigated in Animal Models
}

\author{
Richard F. Lewis \\ Departments of Otolaryngology and Neurology, Harvard Medical School, and \\ Jenks Vestibular Physiology Laboratory, Massachusetts Eye and Ear Infirmary, \\ Boston, Mass., USA
}

\section{Key Words}

Vestibular function · Vertigo · Prosthesis · Implant · Ataxia

\begin{abstract}
Loss of peripheral vestibular function results in debilitating postural, perceptual, and visual symptoms. A new approach to treating this clinical problem is to replace some aspects of peripheral vestibular function with a prosthesis that senses head motion and provides this information to the brain by stimulating the vestibular nerve. In this paper, I review studies done in animals over the past 15 years which lay the groundwork for transferring this approach to human patients with severe peripheral vestibular damage. The animal studies demonstrate that the visual and perceptual defects associated with peripheral vestibular damage can be improved with a vestibular implant, but the data on postural control remain less conclusive at this point in time.

(c) 2015 S. Karger AG, Basel
\end{abstract}

\section{Introduction}

The vestibular regions of the labyrinth transduce angular head velocity in three dimensions (the three semicircular canals) and the vector sum of gravity and the inertial force produced by linear acceleration [gravito-inertial force (GIF), the otolith organs; see Liberman et al. [1] for a review]. The brain is able to use this information to estimate angular head velocity, head orientation relative to gravity, and linear acceleration, and these three signals drive reflexive eye movements produced by head motion [the vestibulo-ocular reflex (VOR)], vestibulospinal projections that contribute to postural control, and ascending projections to 
the thalamus and cerebral cortex that underlie vestibular perception. Patients who lose significant peripheral vestibular function in both ears therefore suffer from postural instability, spatial misperception, and motion of visual images during head movements (oscillopsia). Since the hair cells of the inner ear, a common site of peripheral pathology, do not regenerate, considerable interest has been focused over the past decade on developing vestibular prosthetics [2]. Similar to the cochlear implant, the vestibular implant transduces motion information and provides this to the brain by modulating the rate or current of electrical pulses applied to the canal ampullary nerves. Most of the work over the past decade has been in animal models, but more recently similar technological approaches have been transitioned to human patients with severe vestibular deficits. This paper reviews our experience studying vestibular implants in animal models, work that has helped to set the stage for the current transition to humans.

\section{Basic Structure of the Prosthesis}

It is important to stress that all studies to date have focused on a semicircular canal implant that senses angular head velocity in one or more dimension(s) and transfers this information to the brain by modulating electrical stimulation of the canal ampullary nerve(s). The reason for this focus is that the stereocilia of the hair cells in the canal's cristae are aligned so that head rotation either increases or decreases the firing rate of all primary afferents that innervate the canal, depending on the direction of head rotation. This basic process can be simulated by a canal implant that senses angular head velocity and modulates the strength (rate or amplitude) of the current pulses applied to the canal ampullary nerve. In contrast, the stereocilia in the otolithic maculae are oriented radially and reverse direction at a zone called the striola. The primary otolith afferents therefore modulate in a complex pattern when the head tilts or translates, and this cannot be readily simulated by increasing or decreasing the rate or amplitude of current pulses applied to the otolithic afferents.

The prototype canal implant developed and implemented by Gong and Merfeld [3, 4] consisted of an angular velocity sensor, an electrode implanted near the ampulla of one semicircular canal, and the power and circuitry needed to modulate the rate of biphasic current pulses supplied by the electrode as a function of angular head velocity in the plane of the stimulated canal. To encode head rotation in either direction with a unilateral implant, the tonic rate of stimulation was chosen to be well above the normal tonic discharge rate of canal afferents such that it could modulate up when the head rotated towards the stimulated ear and down when it rotated away from that ear. Although initial studies showed that eye movement responses could be generated by modulating either the amplitude (current) or the rate of the pulses [3], the prototype prosthesis used rate modulation since that recapitulates how the normal canal encodes angular head velocity.

\section{Rodents: Static Vestibular Function}

Several of the basic stimulation features were worked out initially in guinea pig models. It was first demonstrated that the brain compensates for the high tonic rate (250 pulses/s) of stimulation, as evidenced by attenuation of the nystagmus generated by the tone imbalance induced by the supranormal stimulation rate [5]. Two important elements of this basic process (rebalancing of vestibular tone) were subsequently studied. First, there was concern that the eye would move with each biphasic pulse, e.g. it would oscillate at a rate of $250 \mathrm{~Hz}$. Eye movement recordings demonstrated that, indeed, when the stimulation was first turned 
on, the eye did oscillate at this frequency, but relatively rapidly these oscillations resolved [6], presumably through a process of adaptation and/or habituation. Second, there was concern that, in human subjects, turning the high stimulation rate on would engender vertigo; thus, studies were undertaking to determine if repeated exposures to the stimulation on-to-off and off-to-on transition would lead to reduction in the nystagmus (and presumably the vertigo) produced by these transitions [5]. Experiments showed that the amount of nystagmus produced by these transitions rapidly reduced after they were repeated, results that were interpreted as evidence for dual-state adaptation to the stimulation-on and stimulation-off states. Habituation could not be ruled out as a mechanism, however, and so additional studies compared animals for which the stimulation was turned on and off abruptly with animals for which the stimulation was ramped up slowly and then turned off abruptly, thereby matching the total amount of stimulation provided to each set of animals but changing the dynamics of the stimulation-on transition [7]. The results were consistent with adaptation rather than habituation as the principal underlying mechanism. In sum, studies in guinea pigs explored the basic relationship between pulse rate and amplitude, and the evoked eye movements showed that the tone imbalance associated with a high tonic level of electrical stimulation in one ear resolved relatively quickly, which suggested that the primary underlying mechanism for the rebalancing of vestibular tone was adaptation.

\section{Squirrel Monkeys: The One-Dimensional VOR}

To evaluate the capabilities of the unilateral implant to generate VOR responses, it was necessary to shift studies to foveate animals with binocular frontal vision that more closely reflects human eyes. Squirrel monkeys were therefore instrumented with a unilateral, lateral canal implant that sensed head rotation in yaw and stimulated one lateral canal ampullary nerve. An implant on the skull held the velocity sensor, power supply, and prosthesis circuity, and the animal could move freely in its cage during prolonged periods of stimulation that lasted more than a year in some cases [8]. To test the prosthesis, it was first necessary to inactivate the lateral canals, which was accomplished by plugging them bilaterally. This procedure eliminated the yaw VOR over the range of head rotation frequencies we tested (typically sinusoids from 0.01 to $1.0 \mathrm{~Hz}$, and velocity steps). The angular velocity signal transduced by the sensor was high-pass filtered with a cutoff frequency of $0.03 \mathrm{~Hz}$ (consistent with a time constant of $5 \mathrm{~s}$ ) to simulate the high-pass characteristics of the normal semicircular canals. The transfer function used to relate the filtered head velocity to the rate of pulses provided by the implanted electrode was a hyperbolic tangent, a function which is linear over much of its range but saturates at the upper and lower extremes. Using this approach, the baseline stimulation rate was $200-250 \mathrm{~Hz}$ with the head stationary and modulated up or down, based on the direction of head rotation, in an essentially linear manner over the physiologic range of head angular velocities. The slope of the linear portion, whose units were pulses/s/ degrees/s, could be modified to increase or decrease the sensitivity of the implant, and typically slopes near 1 or 2 were employed. Similarly, the time constant of the high-pass filter could also be modified [8].

Using this approach, we studied a number of squirrel monkeys over prolonged periods of stimulation, sometimes cycling their stimulation sensitivity between 1 and 2, and sometimes cycling the time constant of the filter between 5 and $80 \mathrm{~s}$ [9]. We found several fundamental behavioral characteristics of the canal implant with these studies. When stimulation was turned on, a robust VOR response was recorded, but this rapidly attenuated over the first $2 \mathrm{~h}$ with the gain (eye velocity/head velocity) values that approximated the normal VOR (e.g. 0.4 ) to values as low as 0.1 . We intuited that this rapid reduction in eye movement responses 
was generated in the brain by a combination of habituation (reduced response to repetitive stimulation) and adaptation (the brain's attempt to minimize a behavioral error, in this case the tone imbalance that resulted in spontaneous nystagmus). With prolonged stimulation, however, particularly when we cycled between the off state and sensitivities of 1 and 2, we found that the gain gradually increased. Since this increase in gain was associated with an improvement in the rotational axis of the eyes (e.g. the direction of eye rotation shifted to better align with the direction of head rotation during testing in the yaw plane), it appeared that the brain was able to use visual information (retinal slip signals) to parametrically adapt the VOR generated by the prosthesis in a manner that recapitulated the visually guided adaptation of the normal VOR. This key finding was the first indication that the prosthetic rotational cue could engage in normal sensory integration with the visual system. A particularly dramatic example of visually guided adaptation of the prosthesis-generated VOR was observed when the stimulating electrode was moved from the lateral canal to the posterior canal ampulla, with the angular velocity sensor remaining aligned with the lateral canal's sensitive axis [10]. When the animal was rotated in yaw, the prosthesis generated vertical and torsion eye movements because the posterior canal ampullary nerve was activated (increasing retinal image motion) rather than horizontal eye movements that would suppress retinal image motion. Within 1 week, however, the eye movement axis shifted such that yaw rotation of the head produced primarily horizontal eye movements, even though the input to the brain was still through the posterior canal ampullary nerve. These results demonstrated a pronounced central adaptation of the rotational axis of the VOR and confirmed that visual (retinal slip) signals were able to modify the rotational cue provided by the prosthesis in a most dramatic manner.

Although the gain and axis of the VOR were approximately normal in these animals, the phase of the response (timing of eye movements relative to head movements) was markedly abnormal. While the normal VOR shows a moderate phase lead at low frequencies (consistent with a VOR time constant of 15-20 s), the prosthesis-induced VOR showed a much larger phase lead consistent with a time constant as low as $2 \mathrm{~s}$ [8]. This was a particularly confusing observation, since the input provided by the prosthesis had a time constant that was larger than the VOR response. Normally the opposite is observed, since the canal time constant is about $5 \mathrm{~s}$ [1], but a central integrator referred to as velocity storage increases the time constant of the VOR to the 15- to 20 -second range. The time constant can most easily be conceptualized as the rate at which the eye movement response decays when the head is rotated at a constant angular velocity; the decay is fit by a single exponential, and the time constant of the VOR is the time constant of this exponential - a short time constant means the VOR decays rapidly, a long time constant means it decays slowly [11]. While this may seem to be an esoteric concern, it is actually crucial that the prosthetic input engage the velocity storage integrator, since this is the key to the interaction between otolith-mediated GIF information and canal-mediated rotational information, an interaction that underlies the ability of the brain to segregate shifts in GIF into their tilt (gravitational) and translational (inertial) components.

To determine if this critical interaction occurred with the prosthesis input, we used a motion paradigm commonly used to demonstrate this interaction in normal subjects, referred to as postrotational tilt or dumping: the head is rotated at a constant angular velocity about an earth-vertical yaw axis, the rotation is suddenly stopped, and then it is tilted away for the vertical axis. Because of its high-pass filter characteristics, when the head rotation is suddenly stopped in this manner, the canals provide a signal to the brain indicating that the head is rotating in the opposite direction. Tilting the head away from the vertical axis suppresses the postrotational VOR (dumping) and also causes the eye's rotational axis to shift away from the head-centered axis to a gravity-centered axis, i.e. the eye's rotational axis shifts to align with 
Lewis: Vestibular Prostheses Investigated in Animal Models

gravity [11]. Although the prosthesis' VOR time constant was not prolonged in a manner that demonstrates velocity storage, we found that when the lateral canal was stimulated by the prosthesis with the animal's head tilted, the VOR response was attenuated and its rotational axis shifted towards alignment with gravity [12]. This critical observation shows that the prosthesis-mediated angular velocity signal interacts with otolith signals in a qualitatively normal manner and illustrates for the first time that this can be dissociated from the dynamic effects of velocity storage on the low-frequency VOR.

The final study we performed on squirrel monkeys was to examine the linearity of the interaction when both ears were stimulated simultaneously. By implanting electrodes in both lateral canals, we could provide short-term electrical stimulation in both ears simultaneously in a manner such that they either reinforced or suppressed each other. For example, sinusoidal inputs to both ears should summate if they are $180^{\circ}$ out of phase but should cancel if they are in phase. By measuring eye movement responses during these acute experiments, we showed that the brain combines inputs from both ears in an approximately linear manner, since the VOR doubled when the inputs were out of phase and was attenuated when the inputs were in phase [13]. These results indicate that the brain can combine inputs from multiple stimulated canals in an essentially linear manner, which implies that activation of several canals in the same ear should summate in the brain in a manner that recapitulates the summation of inputs from the lateral canals of both ears.

In summary, as our emphasis shifted from static vestibular function to dynamic vestibular function, our experiments shifted from afoveate, lateral-eyed guinea pigs to foveate, frontaleyed squirrel monkeys. In squirrel monkeys we showed that motion-modulated electrical stimulation of the lateral canal ampullary nerve could produce a one-dimensional VOR response that was compensatory in that it served to reduce retinal image motion during head movements. Furthermore, the VOR showed clear evidence of multisensory integration that was qualitatively normal, as both visual and otolith inputs interacted with the prosthesismediated VOR. Finally, we established that prosthesis-mediated eye movements summate in an essentially linear manner, a crucial observation for the development of multidimensional canal prosthetics.

\section{Rhesus Monkeys: Multidimensional Prostheses and Higher-Level Vestibular System-Mediated Behaviors}

Experiments subsequently shifted to rhesus monkeys, since they could be trained on complex behaviors which allowed us to examine the VOR in more detail and to investigate the potential utility of the canal prosthesis in order to improve perception and posture in vestibulopathic subjects. We implemented a three-dimensional canal prosthesis in a subset of our rhesus monkeys, extending the one-dimensional approach so that three angular velocity sensors on the head were aligned with the sensitive axes of the three canals in one ear, and each sensor modulated the rate of stimulation in an electrode implanted in the ampulla of the corresponding canal. We also implemented multisite electrodes at this time, which allow us to provide a current to multiple sites on one electrode and to use a local return on the electrode rather than a remote return. This approach allows us to deliver stimulation more precisely to the ampullary nerve and to constrain current paths such as crosstalk between the different canals.

Vestibulo-Ocular Reflex

We measured the angular VOR in one animal with a three-dimensional canal prosthesis during yaw, roll, and pitch rotation about an earth-horizontal axis and demonstrated a clear 
Lewis: Vestibular Prostheses Investigated in Animal Models

extension of our one-dimensional studies, as compensatory eye movements were generated for each axis of rotation that served to reduce retinal image motion [14]. Future work will involve comparing VOR responses after ablation and during prosthetic stimulation to VOR thresholds [15], which are defined as the smallest angular head velocity that produces an eye movement in the compensatory direction (e.g. the opposite direction of the head rotation). While the gain is determined by the size of the signal provided by the canal or prosthesis, the threshold assays the noise in the peripheral and central vestibular signal, since it is determined by the ratio of the signal to the noise. Understanding how noise can affect prosthetic stimulation may prove to be very important, since it has become clear in other sensory systems that titrating noise to the correct value actually improves the performance of sensory systems through a mechanism known as stochastic resonance [16].

\section{Perception}

Percepts of motion and orientation rarely enter our consciousness, unless they are abnormal and due to damage to the inner ear, 8th cranial nerve, or brain. Motion percepts can be quantified in various ways, including the magnitude of tilt or rotation, and we chose to focus on the size of the perceived head tilt relative to the earth-vertical axis. This may seem like a problematic measure given that we are testing a canal (angular velocity) prosthesis rather than an otolith (GIF) prosthesis, but the brain appears to calculate the orientation of the head relative to gravity by temporally integrating the angular velocity signals supplied by the canals. Indeed, the ability of a canal prosthesis to affect perceived head orientation is a crucial capability, as it implies that the canal prosthesis may be able to improve more complex integrative behaviors such as perception and balance in vestibulopathic subjects rather than simply improving the VOR, which would reduce oscillopsia with head motion. We tested this by first training rhesus monkeys to perform a variant of the subjective visual vertical (SVV) test, which involves rotating a light bar in the absence of other visual cues such that it is perceived to be parallel to gravity. We found that monkeys perform this task in a manner that is qualitatively similar to humans, with perception of gravity based on the frequency of head motion and on the integration of canal and otolith signals [17]. We then tested monkeys on this task after an electrode had been implanted into one posterior canal, and with the head upright, when stimulation was provided, the SVV responses deviated away from the stimulated ear, consistent with a misperception of roll tilt towards the canal that was activated [18]. This key finding suggests that a canal prosthesis can indeed modulate the perceived orientation of the head relative to gravity. A more definitive test would be to measure SVV responses with and without motion-modulated canal stimulation in three dimensions in animals that have severe bilateral vestibular deficits. Our preliminary results performing this experiment suggest that bilateral vestibular damage degrades the perception of head orientation, but that this improves when motion-modulated stimulation is provided. More definitive experiments must be run to verify and extend this finding, but it is an exciting result, because it implies that canal prostheses may improve vestibular system-mediated perceptions in vestibulopathic subjects.

One interesting issue to consider is the effect of providing an angular velocity signal to the brain without a concomitant otolith signal [19]. In a normal subject, if the head rotates around an axis not aligned with gravity, the canals provide the angular velocity signal and the otolith organs measure the shift in the orientation of the GIF. In a patient with severe vestibular damage who is utilizing a canal prosthesis, a similar head rotation would provide a facsimile of the three-dimensional angular velocity signal, but the damaged otolith organs would not provide the appropriate signal indicating a shift in GIF orientation. In this scenario, the brain's estimate of the change in head orientation relative to gravity (the ' $G$ ' in GIF), calculated by integrating the prosthetic velocity signal, would not be accompanied by a shift in the GIF, which would imply that an inertial force must be present (the 'I' in GIF), produced by linear 
acceleration. In other words, the brain may well make a misestimate of linear acceleration in this situation, which would be expected to impair perception and postural stability. Our preliminary results in rhesus monkeys do suggest that an aberrant estimate of translation (as reflected in the eye movement response) is generated by the prosthesis-mediated rotational signal. It is unclear, however, if this situation would persist in patients with chronic loss of peripheral vestibular function (the more relevant situation for patients with vestibular damage), as the linkage between the components of the GIF may degrade when their tight association is chronically absent.

\section{Posture}

Patients with severe vestibular damage are at risk because of falls, which are directly traceable to the loss of vestibular system-mediated postural control. While some fraction of this control is modulated at the vestibulospinal level, it appears that higher-level vestibular processing also contributes to postural control. One potential mechanism [20] is that body orientation relative to gravity is the controlled variable, and this is calculated by adding head orientation relative to gravity (derived from vestibular information) to head-on-body information (derived from neck proprioception primarily). Since our perceptual studies suggest that the first step of this process (head in space) can be accomplished by the prosthesis, it is logical to assume that the prosthesis may similarly be able to help improve the brain's estimate of body in space. Experiments that support this contention, however, are sorely lacking. Our preliminary results suggest that monkeys with severe bilateral vestibular damage can improve postural stability during rapid head turns (which typically destabilize subjects with peripheral vestibular loss), but these results are not definitive and require considerable additional study to substantiate.

\section{Future Directions}

A number of important problems remain to be worked out before it can be concluded that a vestibular implant may be an appropriate way to treat patients suffering from severe vestibular loss. Regarding safety, it appears that hearing is usually relatively preserved after the implantation of canal electrodes [21], but it is less certain if the residual canal function is damaged by the implant. The most effective way to transfer angular head velocity information from the sensor to the brain via electrical stimulation of the ampullary nerve remains unclear, with options including rate, amplitude, or combined rate and amplitude coding of velocity in the electrical pulse characteristics (reviewed in Lewis [19]). Desynchronizing firing in the stimulated nerves and adding small amounts of random neural activity or noise may also benefit the behavioral response, and this could be accomplished by superimposing a veryhigh-frequency depolarizing pulse train on the motion-modulated stimulation rate [22]. The potential beneficial effects of the canal prosthesis must be determined when presented without a concomitant otolith signal, and it may prove to be necessary to implement an otolith prosthesis in addition to a canal prosthesis to obtain the maximal beneficial effect. Finally, the behavioral benefits of a canal or canal/otolith prosthesis must be determined in animal or human subjects suffering from severe bilateral vestibular damage for the field of vestibular implants to progress.

\section{Disclosure Statement}

This work has been funded by the National Institutes of Health and by MED-EL Corporation. 
Lewis: Vestibular Prostheses Investigated in Animal Models

\section{References}

1 Liberman MC, Rosowski JJ, Lewis RF: Physiology and pathophysiology; in Merchant SN, Nadol JB (eds): Schuknecht's Pathology of the Ear. Hamilton, BC Decker, 2010, pp 47-76.

-2 Dakin CJ, Elmore LC, Rosenberg A: One step closer to a functional vestibular prosthesis. J Neurosci 2013;33: 14978-14980.

-3 Gong W, Merfeld DM: Prototype neural semicircular canal prosthesis using patterned electrical stimulation. Ann Biomed Eng 2000;28:572-581.

4 Gong W, Merfeld DM: System design and performance of a unilateral horizontal semicircular canal prosthesis. IEEE Trans Biomed Eng 2002;49:175-181.

5 Merfeld DM, Gong W, Morrissey J, Saginaw M, Haburcakova C, et al: Acclimation to chronic constant-rate peripheral stimulation provided by a vestibular prosthesis. IEEE Trans Biomed Eng 2006;53:2362-2372.

6 Saginaw MA, Gong W, Haburcakova C, Merfeld DM: Attenuation of eye movements evoked by a vestibular implant at the frequency of the baseline pulse rate. IEEE Trans Biomed Eng 2011;58:2732-2739.

7 Lewis RF, Nicoucar K, Gong W, Haburcakova C, Merfeld DM: Adaptation of vestibular tone studied with electrical stimulation of semicircular canal afferents. J Assoc Res Otolaryngol 2013;14:331-340.

$>8$ Merfeld DM, Haburcakova C, Gong W, Lewis RF: Chronic vestibule-ocular reflexes evoked by a vestibular prosthesis. IEEE Trans Biomed Eng 2007;54:1005-1015.

-9 Lewis RF, Haburcakova C, Gong W, Makary C, Merfeld DM: Vestibuloocular reflex adaptation investigated with chronic motion-modulated electrical stimulation of semicircular canal afferents. J Neurophysiol 2010;103: 1066-1079.

10 Lewis RF, Gong W, Ramsey M, Minor L, Boyle R, et al: Vestibular adaptation studied with a prosthetic semicircular canal. J Vestib Res 2002-2003;12:87-94.

-11 Raphan T, Cohen B: Velocity storage and the ocular response to multidimensional vestibular stimuli. Rev Oculomot Res 1985;1:123-143.

12 Lewis RF, Haburcakova C, Gong W, Karmali F, Merfeld DM: Spatial and temporal properties of eye movements produced by electrical stimulation of semicircular canal afferents. J Neurophysiol 2012;108:1511-1520.

-13 Gong W, Haburcakova C, Merfeld DM: Vestibulo-ocular responses evoked via bilateral electrical stimulation of the lateral semicircular canals. IEEE Trans Biomed Eng 2008;55:2608-2619.

14 Lewis RF, Haburcakova C, Gong W, Lee D, Wall C 3rd, et al: Vestibular prosthesis tested in rhesus monkeys. Conf Proc IEEE Eng Med Biol Soc 2011;2011:2277-2279.

15 Haburcakova C, Lewis RF, Merfeld DM: Frequency dependence of vestibuloocular reflex thresholds. J Neurophysiol 2012;107:973-983.

16 Collins JJ, Chow CC, Imhoff TT: Stochastic resonance without tuning. Nature 1995;376:236-238.

-17 Lewis RF, Haburcakova C, Merfeld DM: Roll tilt psychophysics in rhesus monkeys during vestibular and visual stimulation. J Neurophysiol 2008;100:140-153.

-18 Lewis RF, Haburcakova C, Gong W, Lee D, Merfeld D: Electrical stimulation of semicircular canal afferents affects the perception of head orientation. J Neurosci 2013;33:9530-9535.

19 Lewis RF: Advances in the diagnosis and treatment of vestibular disorders: psychophysics and prosthetics. J Neurosci 2015;35:5089-5096.

20 Stapley PJ, Ting LH, Kuifu C, Everaert DG, Macpherson JM: Bilateral vestibular loss leads to active destabilization of balance during voluntary head turns in the standing cat. J Neurophysiol 2006;95:3783-3797.

21 Bierer SM, Ling L, Nie K, et al: Auditory outcomes following implantation and electrical stimulation of the semicircular canals. Hear Res 2012;287:51-56.

22 Rubinstein JT, Wilson BS, Finley CC, Abbas PJ: Pseudospontaneous activity: stochastic independence of auditory nerve fibers with electrical stimulation. Hear Res 1999;127:108-118. 